\title{
Temporal trends in use of tests in UK primary care, 2000-15: retrospective analysis of $\mathbf{2 5 0}$ million tests
}

In this paper by O'Sullivan and colleagues $(B M J$

2018;363:k4666, doi:10.1136/bmj.k4666, 28 November 2018), the authors would like to add the following acknowledgement:

This paper presents independent research funded by the National
Institute for Health Research School for Primary Care Research (NIHR SPCR). The views expressed are those of the authors and not necessarily those of the NHS, NIHR, or Department of Health. 